\title{
Dileptons, hadronic resonances and chiral symmetry - established connections and missing links
}

\section{Stefan Leupold*}

GSI, Darmstadt

E-mail: s.leupold@gsi.de

\section{Markus Wagner}

Institut für Theoretische Physik, Giessen University, Germany

The crucial role of hadronic resonances for the dilepton production and for the transition to the chirally symmetric quark-gluon plasma is pointed out. Furthermore it is stressed that a better understanding of the intrinsic structure of resonances is an important ingredient to gain deeper quantitative insights into chiral symmetry breaking and its restoration.

Critical Point and Onset of Deconfinement - 4th International Workshop

July 9 - 13, 2007

Darmstadt, Germany

\footnotetext{
* Speaker.
} 


\section{Chiral Symmetry Breaking}

As an introduction let me start with the question: How do we know that chiral symmetry is broken? To set the stage let's first look at a symmetry which is not spontaneously broken (except for a small explicit breaking): the isospin symmetry based on the group $S U_{V}(2)$. A continuous symmetry based on such a non-Abelian group demands the existence of multiplets, i.e. degenerate states. Much celebrated examples are e.g. the iso-doublet $(p, n)$ or the iso-triplet $\left(\pi^{+}, \pi^{0}, \pi^{-}\right)$. However, the symmetry tells much more than simply the degeneracy of single-body states. Whole spectra corresponding to different currents must be identical. An isospin transformation turns e.g. the neutral current $j_{V}^{\mu}=\frac{1}{2}\left(\bar{u} \gamma^{\mu} u-\bar{d} \gamma^{\mu} d\right)$ into the charged one $\bar{u} \gamma^{\mu} d$. The spectral information contained in the former current is available from the reaction $e^{+} e^{-} \rightarrow$ hadrons, provided one properly subtracts the isoscalar contributions. On the other hand, the spectral information contained in the charged current is accessible via tau decays. Indeed, to a very good approximation both spectra show the same structure (cf. e.g. [1]). Next let us turn to the chiral symmetry with respect to $S U_{A}(2)$. Transformations turn the vector-isovector current $j_{V}^{\mu}$ into the axial-vector-isovector current $\vec{j}_{A}^{\mu}=\bar{q} \vec{\tau} \gamma_{5} \gamma^{\mu} q$. The consequence of a chirally symmetric world would be, that the corresponding spectra should be degenerate. Both spectra can be studied in tau decays. The weak process $\tau \rightarrow v_{\tau}+$ hadrons involves the difference $j_{V}^{\mu}-j_{A}^{\mu}$. On account of the different G-parity of $j_{V}^{\mu}$ and $j_{A}^{\mu}$ one has access on both spectra separately. The results are shown in Fig. 1. Obviously,
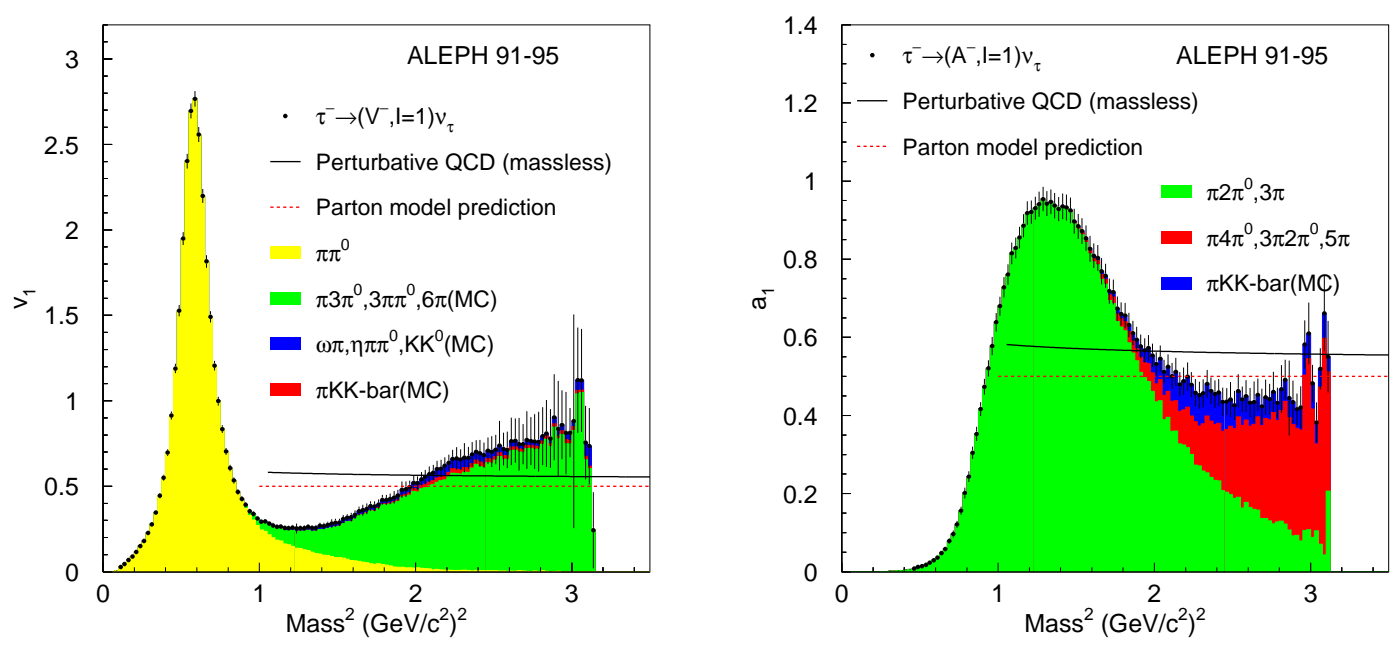

Figure 1: Spectral information contained in the vector-isovector current (1.h.s. [1]) and in the axial-vectorisovector current (r.h.s. [1]).

the spectra are not the same - not even approximately. (Recall that chiral symmetry is explicitly broken by the finite quark masses.) One can see that even more clearly by looking at the difference of the two spectra as shown in Fig. 2. This indicates that chiral symmetry is spontaneously broken.

Typically spontaneous symmetry breaking is lifted at some temperature/density. (The classical example is the ferro magnet with its broken rotational invariance. The symmetry is restored at the Curie temperature.) We expect that this happens also for the chiral symmetry. Thus, at the 


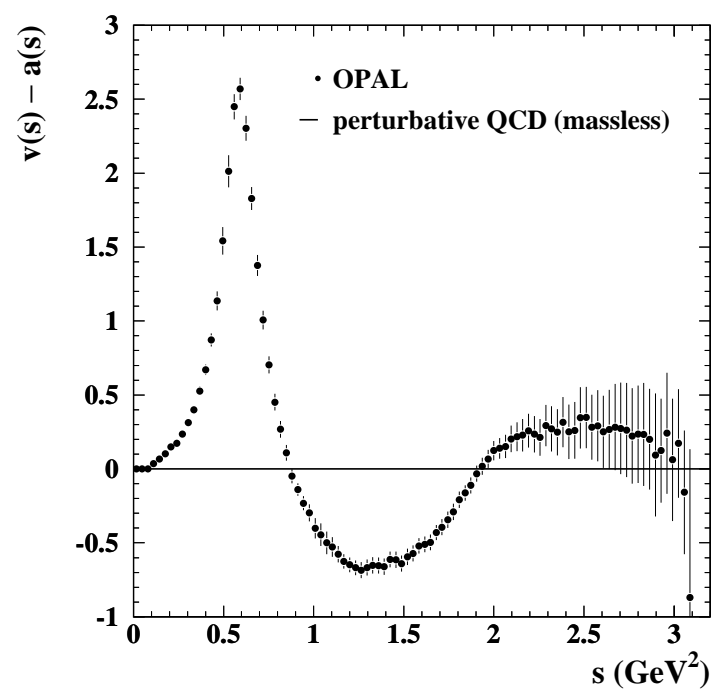

Figure 2: One of the clearest signs of chiral symmetry breaking: The difference of the vector and axialvector spectra [2].

point of chiral restoration we must find the same (in-medium) spectral information in the vector and in the axial-vector channel. From Figs. 1 and 2 we recall how different the spectra are in vacuum. Consequently we expect drastic in-medium changes in both spectra to accommodate for the demand that they become the same at the restoration temperature/density. One should be aware of the fact that this is a qualitative expectation. Quantitatively we (still) need models to describe these changes due to our present lack of understanding of non-perturbative QCD.

Recalling that the vector current couples to dileptons we come to the first connection between chiral symmetry, dileptons and resonances: We expect qualitatively that the dilepton spectrum should drastically change in a medium on account of chiral restoration.

\section{Chiral Restoration and Hadronic Resonances}

As a next step let me recall an interesting relation between chiral restoration and deconfinement. Lattice QCD tells us that (at least for vanishing net baryon density) three phenomena occur at the same temperature [3]: The pressure drastically rises (i.e. the number of the relevant degrees of freedom changes), the two-quark condensate drastically drops (the corresponding susceptibility peaks) and the order parameter of deconfinement, the Polyakov loop also rises. These changes are displayed in Fig. 3 (for more recent figures cf. the talk by F. Karsch).

Let us see whether one can get insight into these coincidences from the low-temperature hadronic point of view: At (very) low (particle or energy) densities a strongly interacting system can be described by a free gas of (stable) hadrons. The reason is that all interactions come with higher powers in the particle densities. At higher densities, of course, interactions or in other words correlations become important. Obviously strong correlations occur whenever hadronic resonances are formed. Thus one might approximate the strongly interacting system at higher densities by a free gas of resonances. (What we describe here is, of course, the much celebrated virial expansion 

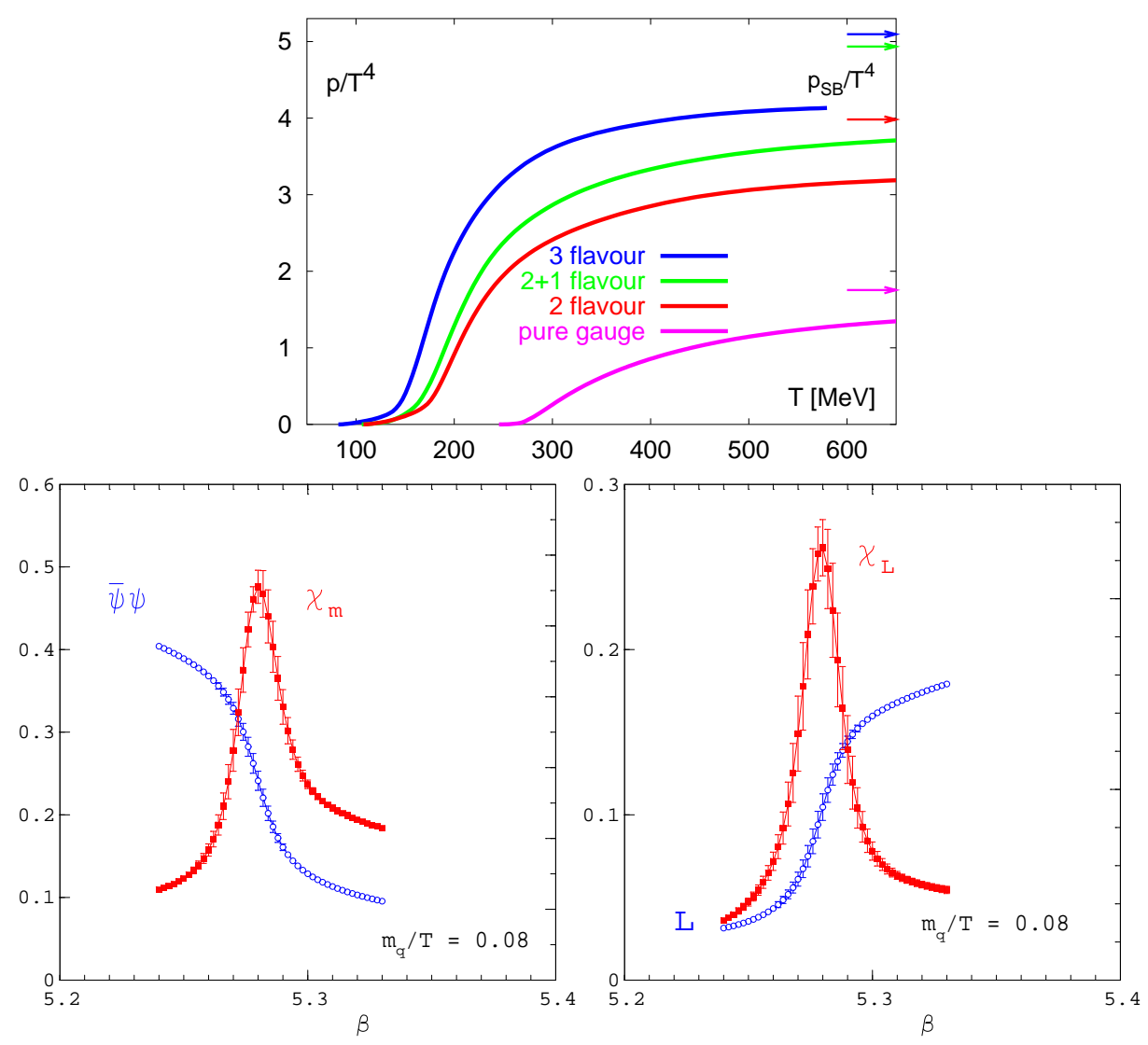

Figure 3: Lattice results for the pressure (top), the two-quark condensate (bottom, left) and the Polyakov loop (bottom, right) [3]. Note that the temperature scales with the lattice parameter $\beta$ (especially $\beta$ should not be mistaken for the inverse temperature).

and the resonance approximation to it [4].) On the other hand, the hadronic resonances (and the stable hadrons) are composite objects and not point-like. Thus, at higher densities the hadrons occupy a large fraction of the available volume. This suggests a percolation transition to take place: As soon as there is enough overlap between the hadrons, quarks cannot decide anymore to which hadron they belong and can move more or less freely without getting outside of the occupied volume [5]. Such a scenario would explain why the liberation of quarks and gluons (which leads to the rise in the pressure and in the Polyakov loop) happens at the same temperature as the chiral restoration.

Next one should ask whether one can find quantitative indications for such a scenario to be true. Indeed, as shown in [6], lattice results for thermodynamic quantities below the transition temperature can be reproduced very well by a free resonance gas (cf. Fig. 4, 1.h.s.). It might appear, however, that thermodynamic quantities are simply not decisive enough. There is still the possibility that the resonance gas approximation is inappropriate for quantities specifically related to the chiral symmetry restoration. Hence, one has to look at order parameters of chiral symmetry breaking. Two such order parameters will be studied in the following, namely the two-quark condensate and a specific four-quark condensate which is related to the second moment of the difference $v-a$ 

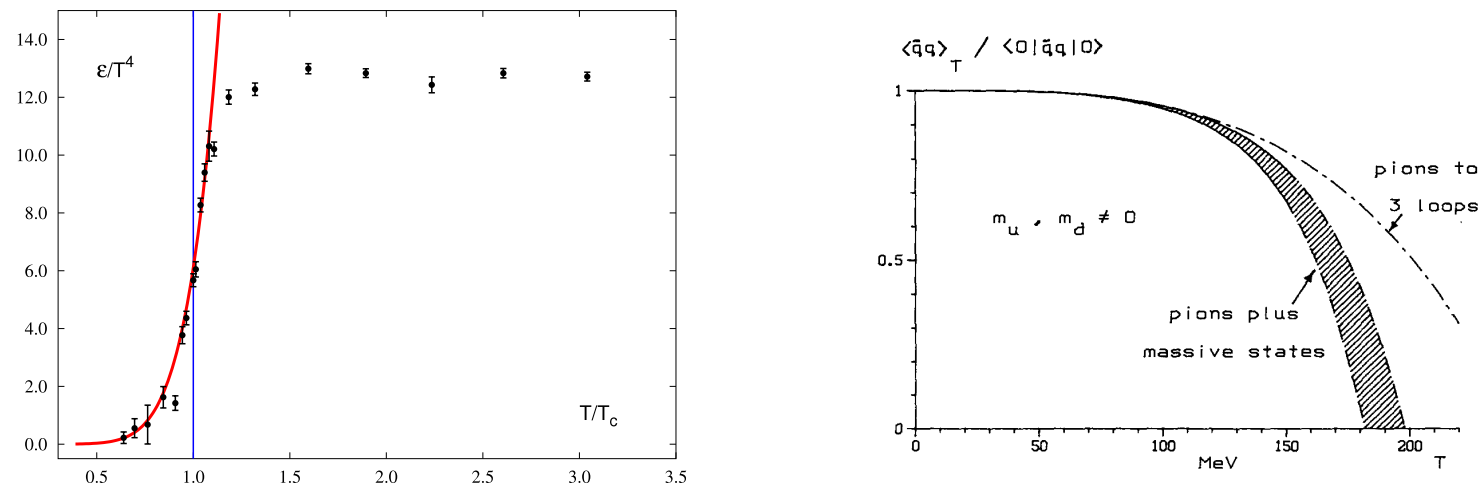

Figure 4: L.h.s.: Comparison of lattice results and a free resonance gas [6]. R.h.s.: Drop of the two-quark condensate using chiral perturbation theory for interacting pions and in addition a free resonance gas [7].

depicted in Fig. 2 [8]:

$$
\int_{0}^{s_{0}} d s s^{2}[v(s)-a(s)]=\left\langle\mathscr{O}_{4 \mathrm{q}}\right\rangle_{\mu=\sqrt{s_{0}}} \quad \text { for sufficiently large } s_{0} .
$$

The temperature dependence of the two-quark condensate has been investigated already some time ago in [7]. It has been demonstrated that an interacting pion gas (described by chiral perturbation theory) leads to a drop of the condensate. The effect is, however, not strong enough. In contrast, the sheer presence of non-interacting hadron resonances (together with the pion gas) is sufficient to obtain a very reasonable transition temperature in the range of 180 to $200 \mathrm{MeV}$. This is demonstrated in Fig. 4, right panel. This provides a further indication that the resonance gas scenario is reasonable. Still one might argue that also the two-quark condensate is not specific enough since it is directly related to a thermodynamic quantity, the pressure $p:\langle\bar{q} q\rangle \sim \frac{\partial p}{\partial m_{q}}$.

A completely different order parameter is provided by the four-quark condensate which appears on the r.h.s. of (2.1). Obviously the difference $v-a$ which appears on the 1.h.s. of (2.1) provides an order parameter for chiral symmetry breaking: As discussed in Sec. 1 it has to vanish in a chirally restored system. In [8] the dependence of the four-quark condensate on temperature $T$ and baryo-chemical potential $\mu$ is investigated within a resonance gas approximation. Results are shown in Figs. 5 and 6 . Especially in Fig. 6 the line where the four-quark condensate vanishes is depicted. A word of caution is in order here: At low temperatures (and chemical potentials on the order of the nucleon mass) the hadron gas reduces to a gas of nucleons. Here the results are not trustable any more. At low temperatures the dominant correlations are nucleon-nucleon correlations and not hadronic resonances. Thus the whole picture breaks down. The results of Fig. 6 are reasonable, however, at not too low temperatures (a conservative estimate might be $T>100 \mathrm{MeV}$ ). Indeed, one gets a very reasonable value for the transition temperature at $\mu=0$ (cf. the talks by F. Karsch and S. Fodor). Fig. 6, right panel reveals another very interesting aspect: The transition basically happens at a fixed energy density independent of the net baryon density. (The deviation from this behavior happens at high baryon densities. This area corresponds to very low temperatures where the resonance gas approximation becomes invalid, as discussed before.) Thus the energy density appears to be the control parameter of the transition. 

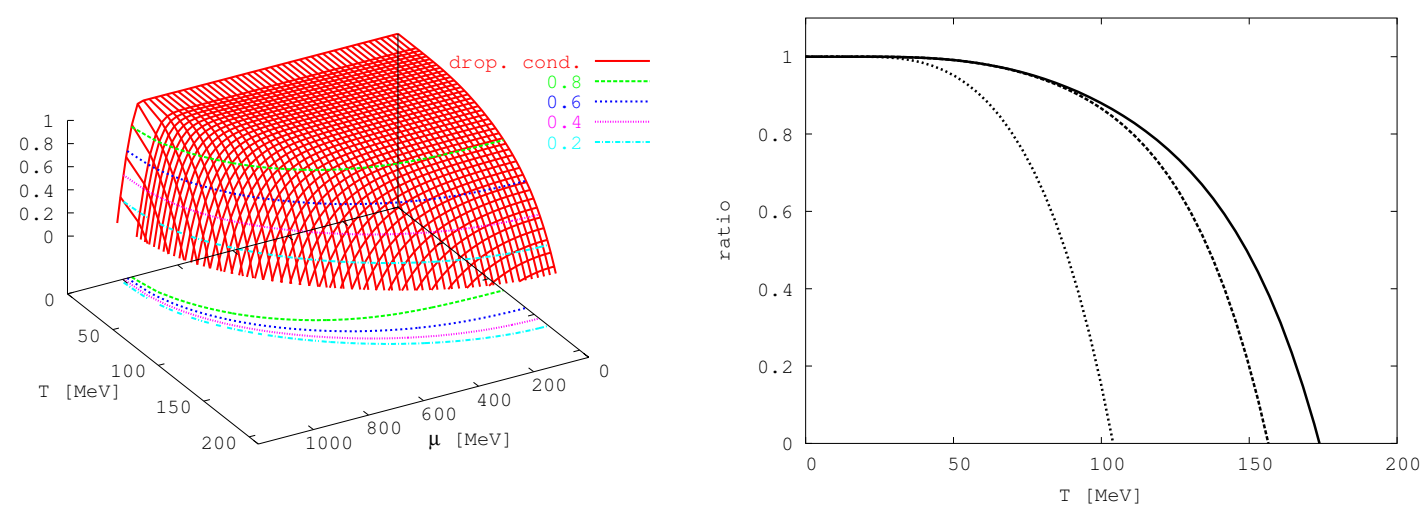

Figure 5: Ratio of in-medium to vacuum four-quark condensate as a function of temperature $T$ and baryochemical potential $\mu$. L.h.s.: 3 -d plot. R.h.s.: Cuts for fixed chemical potentials $(\mu=0,400,800 \mathrm{MeV}$ from right to left) [8].
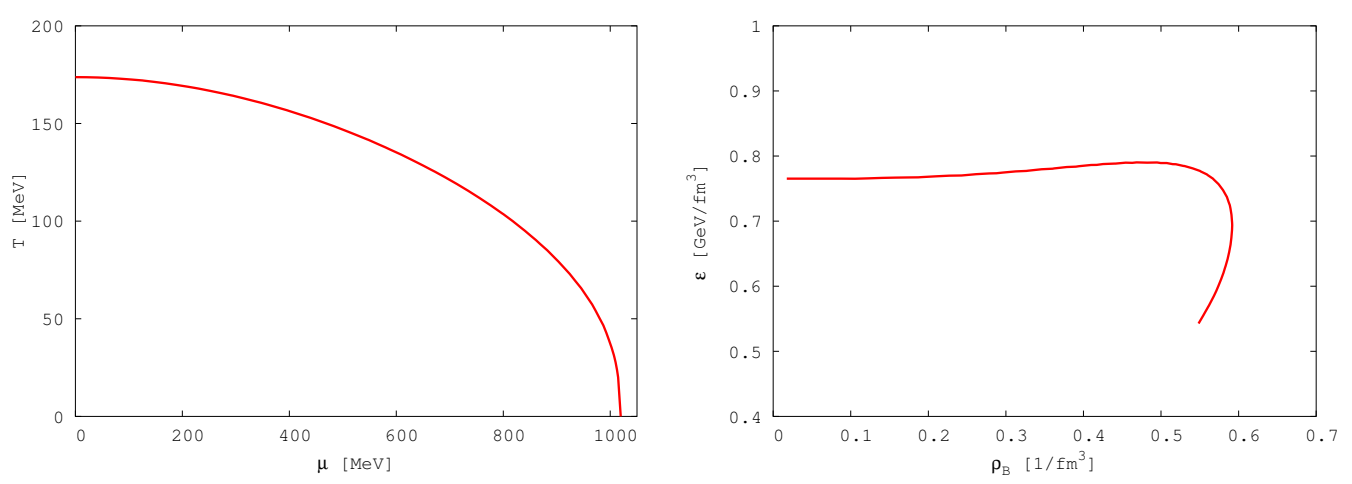

Figure 6: Vanishing of the four-quark condensate in the $T-\mu$ plane (left panel) and in the $\varepsilon$ - $\rho$ plane (right panel) with the energy density $\varepsilon$ and the net baryon density $\rho$ [8].

All these results indicate that the resonance gas approximation yields a reasonable description of a strongly interacting system below the transition to the quark-gluon plasma (for not too low temperatures). Since it deals with hadronic degrees of freedom only, it is clear that it does not work above the transition point. One sees the deviation already in Fig. 4. The same is true for the order parameters: Within the resonance gas approximation the order parameters would not level off or stay at zero as they should, but drop further to negative values. Therefore, we can at best expect that the resonance gas approximation describes the system until close to the transition point, but it does not describe the transition itself.

To summarize this section we find the second connection between chiral symmetry, dileptons and resonances: The resonance gas approximation describes the way to chiral restoration.

\section{Dileptons and Hadronic Resonances}

Having seen that the resonance gas approximation works well for bulk properties and for chiral 

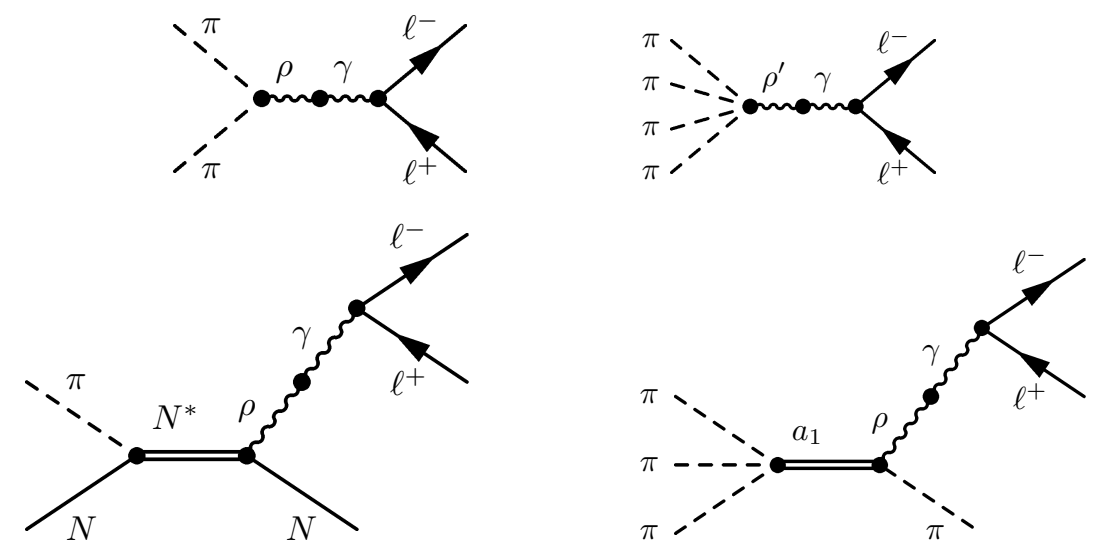

Figure 7: Four generic processes for dilepton production which enter e.g. in-medium hadronic many-body calculations. $\ell^{ \pm}$denote dileptons.

order parameters, we can ask about the importance of resonances for the dilepton production. Here we have to consider the resonances which couple directly to dileptons, but also the Dalitz decays of resonances. To understand the differences and similarities let us look at four generic processes depicted in Fig. 7 which are driven by resonances. From a conceptual/technical point of view one would group together the processes shown in the first line on the one hand side and the processes of the second line on the other hand side. Indeed, for the first group the reaction probabilities can be inferred from the measured back reactions in vacuum. In addition, the total invariant mass of the hadrons translates to the invariant mass of the dilepton pair. In contrast, for the Dalitz decay type processes of the second group part of the available energy is taken away by the hadron which accompanies the dilepton. Therefore such processes populate the low-mass dilepton region. Indeed, in many-body calculations (e.g. [9]) these processes are responsible for the low-mass enhancement. (A full quantitative description might require to iterate these processes as self energies in a Dyson-Schwinger framework.)

Technically, the dilepton production can be related in an equilibrated medium to the dilepton annihilation (i.e. for the following remarks the processes of Fig. 7 have to be read from right to left). The annihilation can be calculated within a virial expansion (cf. e.g. [10]) or, using vector meson dominance, within a spectral function approach (cf. e.g. [11, 12]). The trivial part of such calculations are indeed the vacuum reactions shown in the first line of Fig. 7. Corrections come from the diagrams shown in the second line where the dileptons scatter on one medium constituent. For that reason many people regard the processes of the first line as trivial in-medium effects and the ones from the second line as non-trivial. On the other hand, from the point of view of dilepton production such a distinction is questionable: After all, both processes on the 1.h.s. of Fig. 7 are two-body reactions. One is as trivial as the other and both are actually genuine in-medium processes since they have pions in the entrance channel. From that point of view the only difference is that the importance of the reaction $\pi+\pi \rightarrow \ell^{+} \ell^{-}$can be judged from the measured back reaction while the reaction $\pi+N \rightarrow \ell^{+} \ell^{-}+N$ is not sufficiently known. As already pointed out such processes are regarded as responsible for the low-mass enhancement. This should be motivation enough to measure the reaction $\pi+N \rightarrow \ell^{+} \ell^{-}+N$ as accurate as possible to constrain or crosscheck the elementary input which enters hadronic many-body calculations. 
While the processes on the 1.h.s. of Fig. 7 are two-body reactions, the diagrams on the r.h.s. display rather non-trivial many-body reactions. Clearly the role of such processes can only be assessed in heavy-ion processes. The importance of the process $4 \pi \rightarrow \ell^{+} \ell^{-}$for the dilepton spectrum above $1 \mathrm{GeV}$ has been stressed recently in [9]. A second aspect which also is especially relevant for heavy-ion physics is related to unitarity: A $\rho$-meson which is produced (as an intermediate state) e.g. in the reaction $\pi+\pi \rightarrow \rho$ knows about the other production mechanisms (e.g. $\pi+N \rightarrow \rho+N$ displayed in Fig. 7, bottom, left). A proper treatment leads to a depletion of the dilepton spectrum around the $\rho$-meson peak. Actually, the whole issue of many-body reactions and unitarity would provide material for a second talk. Therefore, I will stop here and refer to [13, 14].

Obviously, the issue of resonances becomes more involved when studying dilepton production. Nonetheless, we have again seen the important role of resonances which leads us to the third connection between chiral symmetry, dileptons and resonances: The resonances cause the lowmass enhancement in the dilepton spectrum.

\section{Vacuum Properties of Hadronic Resonances — the $a_{1}$-Meson}

For a deeper understanding of dilepton production one key ingredient are definitely the vacuum properties of resonances which decay into dileptons (either directly or via Dalitz decays). The most elementary (and probably the most interesting) question one can ask is: What is the nature of a given resonance? Is it merely of molecule type made out of other hadrons or is it a quarkantiquark or three-quark state, respectively. These questions are lively discussed in the literature for several hadronic resonances (see e.g. [15]). Chiral symmetry breaking plays an important role in the molecule calculations by constraining the possible interactions between the hadronic constituents.
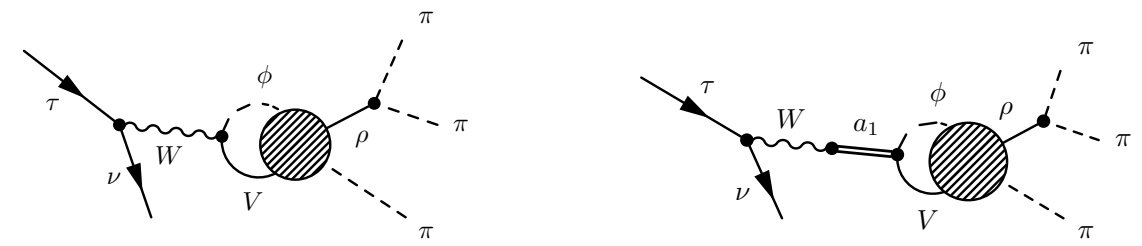

Figure 8: L.h.s.: Coupling of the weak current to two-particle states which are subject to final state interaction (indicated by the blob). R.h.s.: Direct coupling of the weak current to an elementary $a_{1} . \phi$ denotes a Goldstone boson which can be $\pi$ or $K$ while $V$ can be $\rho$ or $K^{*}$.

In the following I will not talk about arbitrary resonances anymore, but concentrate on a particular one, namely the $a_{1}$-meson. The reason is that there exist excellent data for this meson. It appears as a broad structure in the decay $\tau \rightarrow v_{\tau}+3 \pi$ which is free of hadronic initial state interactions (cf. Fig. 1, right panel). With such a clean reaction at hand one has an excellent framework to study the conjecture that the $a_{1}$ is dynamically generated, i.e. a coupled-channel meson-molecule (as suggested for the first time in [15]). This issue has been addressed in a recent work with M. Wagner [16]. We have tested the possibility that the $a_{1}$ is a quark-antiquark state against the scenario that the $a_{1}$ is dynamically generated. We have found strong indications that the latter is true. In this scenario the dominant role is played by the coupling of the weak current to $\pi \rho$ (and to some extent to $K K^{*}$ ). The $a_{1}$-bump which appears in the data is caused by the final state interaction between these two-body states (cf. Fig. 8, 1.h.s.). The strength of this final state interaction 
is fixed in lowest order of the involved momenta by chiral symmetry breaking. It is the famous Weinberg-Tomozawa (WT) interaction. Following [15] for the description of the final state interaction, it turns out that there is only one free parameter in the calculation. This parameter regulates the entrance loop $W \rightarrow \phi V$ where $\phi$ denotes the Goldstone boson $\pi$ or $K$ and $V$ the vector meson $\rho$ or $K^{*}$ (cf. Fig. 8, 1.h.s.). Fig. 9, left panel shows the results of such a calculation varying the single free parameter $\mu_{2}$ (for details see [16]). Obviously one obtains a reasonable description of the data in view of the fact that the one free parameter adjusts the height and the width of the curve at the same time. (The peak position is fairly insensitive to $\mu_{2}$.) A close to perfect agreement with the data can be obtained, if the WT interaction is supplemented by higher order terms in the chiral expansion [16].
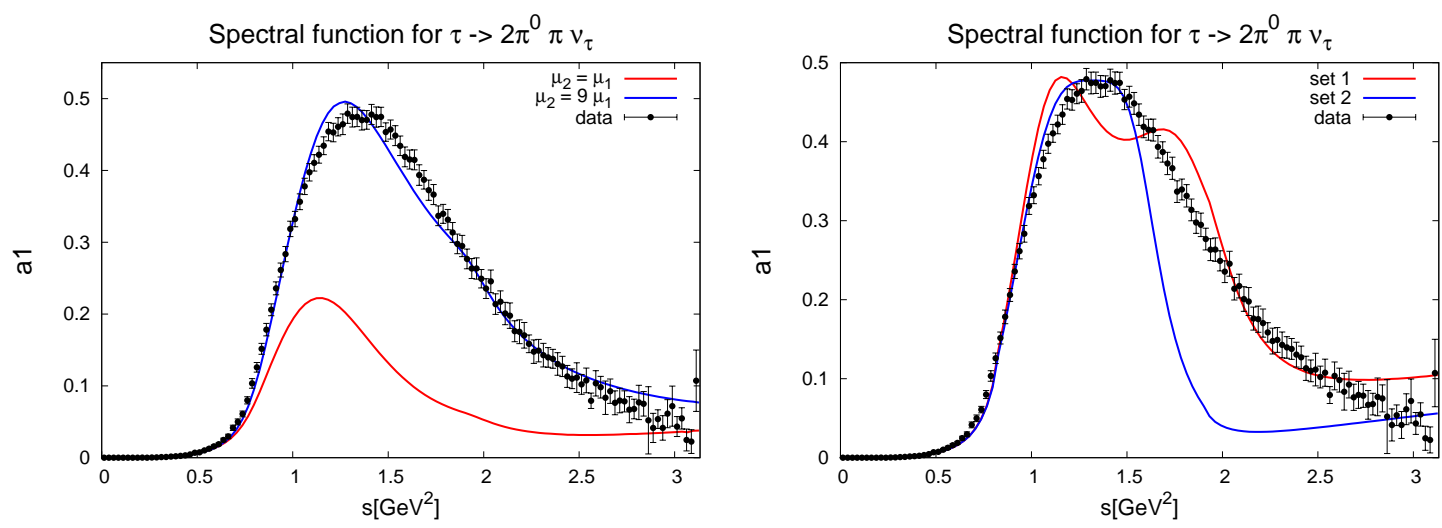

Figure 9: Description of the reaction $\tau \rightarrow v_{\tau}+3 \pi$ with a dynamically generated $a_{1}$ based on the WeinbergTomozawa (WT) interaction (l.h.s.) and with an elementary $a_{1}$ field (r.h.s.). Note that the WT interaction is also included for the second case.

In contrast to this good agreement the inclusion of an elementary $a_{1}$ (i.e. considering both processes of Fig. 8) worsens the result as demonstrated in Fig. 9, r.h.s. It is important to stress that also for these calculations the final state WT interaction is included, since it is a prediction from chiral symmetry and there is no obvious reason why it should vanish in the considered energy range. However, we have used the freedom to choose $\mu_{2}$ to minimize the influence of the WT interaction. Still it is so strong that typically a double hump structure appears (set 1 in Fig. 9, r.h.s.). Only if the additional parameters of the elementary $a_{1}$ are fine-tuned one can obtain a one-peak structure (set 2) - with a wrong width, however. We conclude that the tau decay data suggest that the $a_{1}$ is a coupled-channel meson-molecule.

Our study of the $a_{1}$-meson - where the Weinberg-Tomozawa interaction plays a central role - leads to the fourth connection between chiral symmetry, dileptons and resonances: The nature of some (or even many?) resonances is dictated by chiral symmetry breaking.

\section{Summary and Outlook}

To summarize we have shown four connections between chiral symmetry, dileptons and hadronic resonances. They are marked by bold letters throughout the text and we do not repeat them here.

As an outlook we note that one has to look for further justifications for the resonance gas scenario. In addition, it would be interesting to develop models for the chiral restoration which de- 
scribe also the transition itself and not only the way towards it (as the resonance gas approximation does since it contains only hadronic degrees of freedom more or less fixed to their vacuum properties). In that context we note, however, that the inclusion of the chiral restoration is pretty simple in schematic models which do not have the plethora of degrees of freedom, but it is fairly complicated for realistic models. Also in that context it is, of course, important to figure out which resonances are dynamically generated and which are more elementary quark-antiquark or three-quark states, respectively.

\section{References}

[1] S. Schael et al. [ALEPH Collaboration], Branching ratios and spectral functions of tau decays: Final ALEPH measurements and physics implications, Phys. Rept. 421 (2005) 191 [hep-ex/0506072].

[2] K. Ackerstaff et al. [OPAL Collaboration], Measurement of the strong coupling constant alpha(s) and the vector and axial-vector spectral functions in hadronic tau decays, Eur. Phys. J. C 7 (1999) 571.

[3] F. Karsch, Lattice QCD at high temperature and density, Lect. Notes Phys. 583 (2002) 209 [hep-lat/0106019].

[4] R. Dashen, S. K. Ma and H. J. Bernstein, S Matrix formulation of statistical mechanics, Phys. Rev. 187 (1969) 345.

[5] T. Celik, F. Karsch and H. Satz, A Percolation Approach To Strongly Interacting Matter, Phys. Lett. B 97 (1980) 128.

[6] F. Karsch, K. Redlich and A. Tawfik, Hadron resonance mass spectrum and lattice QCD thermodynamics, Eur. Phys. J. C 29 (2003) 549 [hep-ph/0303108].

[7] P. Gerber and H. Leutwyler, Hadrons Below the Chiral Phase Transition, Nucl. Phys. B 321 (1989) 387.

[8] S. Leupold, Four-quark condensates and chiral symmetry restoration in a resonance gas model, J. Phys. G 32 (2006) 2199 [hep-ph/0604058].

[9] H. van Hees and R. Rapp, Comprehensive interpretation of thermal dileptons at the SPS, Phys. Rev. Lett. 97 (2006) 102301 [hep-ph/0603084].

[10] J. V. Steele, H. Yamagishi and I. Zahed, Dilepton and photon emission rates from a hadronic gas. II, Phys. Rev. D 56 (1997) 5605 [hep-ph/9704414].

[11] M. Post, S. Leupold and U. Mosel, Hadronic spectral functions in nuclear matter, Nucl. Phys. A 741 (2004) 81 [nucl-th/0309085].

[12] R. Rapp and J. Wambach, Chiral symmetry restoration and dileptons in relativistic heavy-ion collisions, Adv. Nucl. Phys. 25 (2000) 1 [hep-ph/9909229].

[13] J. Knoll, Transport dynamics of broad resonances, Prog. Part. Nucl. Phys. 42 (1999) 177 [nucl-th/9811099].

[14] S. Leupold, Trivial and non-trivial in-medium effects, talk given at the "Discussion Forum: What do we learn from dilepton measurements in heavy-ion collisions?", Feb. 27, 2007, GSI, Darmstadt, http://www.gsi.de/documents/DOC-2007-Mar-19-1.pdf.

[15] M. F. M. Lutz and E. E. Kolomeitsev, On meson resonances and chiral symmetry, Nucl. Phys. A 730 (2004) 392 [nucl-th/0307039].

[16] M. Wagner and S. Leupold, $\tau$ decay and the structure of the $a_{1}$, arXiv:0708.2223 [hep-ph]. 